\section{Comparación de los niveles de actividad física medidos con cuestionario de autorreporte (IPAQ) con medición de acelerometría según estado nutricional}

\author{
MIQUEL MARTORELL ${ }^{1 *}$, ANA MARÍA LABRAÑA ${ }^{1 *}$, \\ KARINA RAMÍREZ-ALARCÓN ${ }^{1 *}$, XIMENA DÍAZ-MARTÍNEZ ${ }^{2}$, \\ ALEX GARRIDO-MÉNDEZ ${ }^{3}$, FERNANDO RODRÍGUEZ-RODRÍ- \\ GUEZ $^{4}$, IGOR CIGARROA ${ }^{5}$, JAIME VÁSQUEZ ${ }^{6}$, YENY CONCHA ${ }^{7}$, \\ MARÍA ADELA MARTÍNEZ-SANGUINETTI ${ }^{8}$, ANA MARÍA LEI- \\ VA $^{9}$, CRISTIAN ÁLVAREZ ${ }^{10}$, FANNY PETERMANN-ROCHA ${ }^{11}$, \\ CARLOS SALAS-BRAVO ${ }^{13}$, CARLOS CELIS-MORALES ${ }^{11,12,14}$ \\ en representación del Grupo de Investigación ELHOC
}

\section{Comparison between self-reported and device measured physical activity according to nutritional status}

Background: It is unknown if nutritional status could influence the accuracy of self-reported physical activity (PA) levels. Aim: To compare $P A$ measured using the international physical activity questionnaire (IPAQ) and by accelerometry (ActiGraph) according to nutritional status in Chilean adults. Material and Methods: This is an observational cross-sectional study that used information from the GENADIO project carried out in Chile between 2009-2011. The sample consisted of 322 people. PA levels and sitting time were determined through the IPAQ self-report questionnaire and ActiGraph accelerometers (GTM1). The nutritional status was determined according to body mass index (BMI). Results: Compared with the measurement made with accelerometry, the $I P A Q$ self-report questionnaire underestimated the levels of light $P A$, total $P A$ and sedentary time in $-171.5,-54.8$ and $-40.6 \mathrm{~min} /$ day, respectively. However, IPAQ overestimated the levels of moderate $P A$ and vigorous $P A$ in 91.0 and $47.3 \mathrm{~min} /$ day respectively, compared with accelerometry. Compared with normal weight, obese subjects reported higher levels of moderate $P A$ (105.5 and 48.9 min/day, respectively) and vigorous PA (54.1 and 38.3 min/day respectively). Total $P A$ had a greater delta of underestimation between IPAQ and accelerometer measurements in normal weight than in obese subjects (-122.1 vs $-16.5 \mathrm{~min} /$ day, respectively). Conclusions: The measurement of PA by means of a self-report questionnaire was discrepant with its objective measurement with an accelerometer. The discrepancy is even higher in obese people.

(Rev Med Chile 2020; 148: 37-45)

Key words: Accelerometry; Exercise; Obesity; Sedentary Behavior.
'Departamento de Nutrición y Dietética, Facultad de Farmacia, Universidad de Concepción. Concepción, Chile. ${ }^{2}$ Departamento de Ciencias de la Educación, Universidad del Bío-Bío. Chillán, Chile.

${ }^{3}$ Departamento de Ciencias del Deporte y Acondicionamiento Físico, Universidad Católica de la Santísima Concepción.

Concepción, Chile.

${ }^{4}$ Grupo IRyS, Escuela de Educación

Física, Pontificia Universidad Católica de Valparaíso. Valparaíso, Chile.

${ }^{5}$ Escuela de Kinesiología, Facultad de Salud, Universidad Santo Tomás, Chile.

${ }^{6}$ Vicerrectoría de Investigación y

Postgrado, Universidad Católica del

Maule. Talca, Chile.

'Universidad Tecnológica de Chile INACAP,

Chile. Escuela de Kinesiología, Facultad de

Salud, Universidad Santo Tomás, Chile.

IInstituto de Farmacia, Facultad de Ciencias, Universidad Austral de Chile. Valdivia, Chile.

9Instituto de Anatomía, Histología y Patología, Facultad de Medicina, Universidad Austral de Chile, Valdivia, Chile.

${ }^{10}$ Área Prioritaria de Investigación en Calidad de Vida y Bienestar Humano, Departamento de Ciencias de La Actividad Física, Universidad de Los Lagos, Chile. ${ }^{11}$ Institute of Health and Wellbeing, University of Glasgow, Glasgow, United Kingdom.

${ }^{12}$ Laboratorio de Rendimiento Humano, Grupo de Estudio en Educación, Actividad Física y Salud (GEEAFyS). Universidad Católica del Maule. Talca, Chile.

${ }^{13}$ Departamento de Educación Física, Facultad de Educación, Universidad de Concepción. Concepción, Chile. ${ }^{14}$ Centro de Investigación en Fisiología del Ejercicio (CIFE), Universidad Mayor. Santiago, Chile.

*MM, AML y KRA contribuyeron de igual forma a este manuscrito y son considerados primer autor compartido.

Trabajo no recibió financiamiento. Los autores declaran no tener conflictos de interés.

Recibido el 23 de abril de 2019, aceptado el 11 de noviembre de 2019 .

Correspondencia a:

Dr. Carlos Celis-Morales

Institute of Health and Wellbeing, University of Glasgow, Glasgow, United Kingdom.

Carlos.Celis@glasgow.ac.uk 
L a obesidad es una enfermedad multicausal que se caracteriza por una acumulación excesiva de grasa corporal que puede ser perjudicial para la salud ${ }^{1}$. Según un informe de la Organización Mundial de la Salud (OMS), del año 2016, más de 650 millones de personas eran obesas, lo que corresponde a $13 \%$ de la población mundial, y se estima que causó 2,8 millones de muertes en el mundo, y se concluye que esta enfermedad se ha casi triplicado en el mundo desde hace cuatro décadas ${ }^{2}$. En Chile, según la última Encuesta Nacional de Salud 2016-2017, la prevalencia de obesidad en adultos fue de $34,4 \%$ y el sobrepeso fue de $39,8 \%{ }^{3}$. La misma encuesta reporta que las mujeres presentan $38,4 \%$ de obesidad y los hombres $30,3 \%{ }^{3}$. Entre los principales factores causantes de obesidad están los cambios en los estilos de vida de las personas, incluyendo una menor adherencia a la alimentación saludable ${ }^{4}$ y a la disminución de la realización de actividad física $(\mathrm{AF})^{5}$.

Si bien la práctica regular de AF ha sido asociada a un menor riesgo de obesidad en la población, en Chile, la mayor parte de la cuantificación de esta evidencia ha sido generada a partir de instrumentos de autorreporte, como el Cuestionario internacional de actividad física (IPAQ) y el Cuestionario global de actividad física (GPAQ), los cuales presentan una mayor tendencia a sobreestimar conductas saludables como lo es la práctica de AF y, al mismo tiempo, presentan una tendencia a subestimar conductas no saludables como lo es el sedentarismo o tiempo destinado a estar sentado (conducta sedente) ${ }^{6,7}$. Considerando los altos niveles de obesidad que presenta la población chilena, se desconoce si las actuales estimaciones de AF y sedentarismo de la población podrían estar influenciadas por el estado nutricional de las personas ${ }^{3}$. Una forma de responder a esta inquietud sería la determinación de los niveles de AF con instrumentos de mayor precisión, los que ofrecen una cuantificación objetiva de los niveles de AF de la población, como son los acelerómetros ${ }^{6,8}$. Lo anterior es relevante para la futura implementación de métodos objetivos y precisos en la medición de los niveles de AF de la población chilena, considerando que una gran cantidad de la población presenta un estado nutricional de sobrepeso u obesidad, en los cuales se desconoce el nivel de discrepancia entre ambos métodos de medición de niveles de $\mathrm{AF}^{9}$. Por lo anterior, el objetivo de este estudio fue comparar los niveles de AF medidas mediante cuestionario de autorreporte (IPAQ) y con medición por acelerometría (ActiGraph), según estado nutricional en población chilena.

\section{Material y Método}

\section{Diseño del estudio}

Estudio observacional analítico de corte transversal que utilizó información del proyecto GENADIO (Genes, Ambiente, Diabetes y Obesidad) realizado en Chile entre los años 2009 y $2011^{10}$. Este contó con la aprobación de los comités de ética de la Universidad de Chile, Universidad de Concepción y Universidad de Glasgow (Reino Unido). Todos los participantes firmaron su consentimiento informado previo a la recolección de los datos.

La población estuvo compuesta por residentes pertenecientes a la etnia mapuche y no mapuches (caucásicos) de las regiones del Biobío y de Los Ríos, con edades comprendidas entre los 20 y 72 años, sin historial médico de enfermedades metabólicas o cardiovasculares, y que al momento de la evaluación no estuvieran bajo ninguna prescripción de medicamentos. Los participantes fueron reclutados a través de afiches entregados en centros comunitarios, centros de salud familiar (CESFAM), organizaciones sociales en las ciudades de Concepción, Panguipulli, Ralco y Valdivia. Aquellas personas interesadas en participar fueron invitadas a una reunión donde se explicó el estudio en detalle y tuvieron la posibilidad de exponer sus consultas o inquietudes. Aquellos participantes que decidieron participar fueron invitados a una evaluación inicial donde se recolectó información socio-demográfica y se aplicaron cuestionarios para la medición de estilos de vida. En una segunda visita en ayunas, se procedió a tomar una muestra de sangre para la realización de análisis metabólicos, se midieron las características antropométricas, se aplicó el cuestionario de AF y se programó el acelerómetro de AF (ActiGraph). En la tercera visita, 7 días posteriores a la segunda visita, se procedió a recolectar el monitor de AF y entregar un informe con relación al estado nutricional y salud metabólica del participante.

Del universo de 472 personas que cumplieron con los criterios de inclusión y exclusión, 352 participantes completaron el cuestionario de AF 
(es decir, asistieron a la segunda visita) y de estos, 230 participantes presentaron datos válidos en la medición de acelerómetro que fueron incluidos en este estudio. El 51,3\% de los participantes $(\mathrm{n}=242)$ incluidos en el estudio GENADIO no presentaron información de acelerometría, de estos, 17 participantes presentaron problemas técnicos con el acelerómetro (principalmente agotamiento de batería), 39 no aceptaron utilizar el acelerómetro por motivos de incomodidad o rechazo a este tipo de medición, 94 participantes fueron excluidos ya que utilizaron el acelerómetro por $<3$ días a la semana y 92 fueron excluidos, debido a que utilizaron el acelerómetro por $<10$ $\mathrm{h}$ al día (en promedio lo utilizaron $3,7 \mathrm{~h}$ ), considerándose una medición no representativa ${ }^{10}$.

\section{Autorreporte de actividad física y tiempo sedente}

El autorreporte de AF y el tiempo sedente de los últimos 7 días fueron determinados con la versión abreviada del IPAQ ${ }^{11}$. Los datos fueron reportados en minutos por día (min/día) para cada uno de los dominios de AF, y para estimar el total de AF realizada se corrigió el tiempo reportado por su equivalente metabólico (METs), el cual correspondió a 3,3 METs para AF ligera (caminar), 4 METs para AF moderada y 8 METs para AF vigorosa. El total de AF fue reportado como la suma de los METs para cada una de las intensidades y se utilizó la unidad de METS-min/ semana. El tiempo destinado a AF de transporte asociada al andar en bicicleta fue excluido de los análisis, ya que este dominio no puede ser medido con el acelerómetro de movimiento.

\section{Medición objetiva de actividad física y tiempo sedente}

La medición objetiva de los niveles de AF y tiempo sedente se determinó durante 7 días consecutivos mediante acelerómetros de movimiento ActiGraph (ActiTrainer GTM1, LLC, Pensacola, FL, USA), según metodología reportada previamente $^{12}$. La intensidad de la AF fue determinada mediante el algoritmo de Freedson (AF ligera $<1.952 \mathrm{count} / \mathrm{min}$; intensidad moderada 1.952 $5.724 \mathrm{count} / \mathrm{min}$; e intensidad vigorosa $>5.725$ count $/ \mathrm{min})^{13}$. Los niveles de AF y tiempo sedente fueron presentados min/día. Sin embargo, la AF total fue expresada en MET-min/semana empleando los valores para MET usados para el cuestionario de autorreporte IPAQ, de manera de poder comparar los niveles de AF total estimados por ambos métodos.

\section{Estado nutricional}

La evaluación antropométrica se realizó por personal capacitado utilizando protocolos estandarizados $^{12}$. El peso y la talla corporales fueron determinados con una balanza electrónica (TANITA, modelo TBF 300A, USA) y tallímetro (SECA, modelo A800, USA) con una precisión de $100 \mathrm{~g}$ y $1 \mathrm{~mm}$, respectivamente. El IMC fue calculado usando la ecuación de (peso corporal/talla ${ }^{2}$, expresándose en $\mathrm{kg} / \mathrm{m}^{2}$ ) y el estado nutricional se definió mediante los valores de corte de la OMS y se clasificó en tres categorías: normopeso, sobrepeso y obesidad ${ }^{14}$.

\section{Análisis estadísticos}

Todos los análisis fueron realizados con el software STATA v15 MP. La descripción de variables de tipo continua se realizó mediante la presentación de la media y su respectiva desviación estándar y como porcentaje para variables de tipo categórica. La distribución normal de las variables continuas fue determinada mediante la prueba de Anderson-Darling.

Para estimar las diferencias entre cuestionario de autorreporte y acelerometría para las diferentes intensidades de AF y tiempo sedente, se aplicó la prueba de diferencia de medias para muestras pareadas. Los resultados de estos análisis fueron presentados como promedio y sus respectivos intervalos de confianza [95\% IC]. Se utilizó la gráfica de Bland-Altman para ilustrar el nivel de discrepancia entre ambos métodos de evaluación, esta gráfica incluye el delta entre el cuestionario de autorreporte IPAQ y la medición de acelerometría "ActiGraph" en el eje vertical y lo compara con la medición por acelerometría en el eje horizontal, también se presentan 95\% de los rangos de concordancia entre métodos ${ }^{15}$.

Para estimar si hubo una tendencia significativa a sobreestimar o subestimar los niveles de AF según estado nutricional, se aplicaron análisis de regresión lineal. Para estos análisis, la variable de salida correspondió a la variable de AF de interés y la variable de exposición insertada en el modelo estadístico fue la interacción entre el método de evaluación de la AF y el estado nutricional. El valor p para tendencia fue estimado con regresión lineal, pero la variable de salida utilizada correspondió 
al delta entre [IPAQ - acelerómetro] y la variable de exposición utilizada fue estado nutricional codificada como variable ordinal $(0=$ normo peso, $1=$ sobrepeso, 2 = obeso). Diferencias estadísticamente significativas fueron aceptadas con un valor $\mathrm{p}<0,05$.

\section{Resultados}

Las características de los participantes según sexo se muestran en la Tabla 1. En la Figura 1 y Tabla 2 se ilustran las diferencias entre ambos métodos de evaluación de niveles de AF. Para AF de intensidad ligera se observó una subestima-

Tabla 1. Características de los participantes según sexo

\begin{tabular}{|lcc|}
\hline & $\begin{array}{c}\text { Mujeres } \\
(\mathbf{n}=\mathbf{1 4 3})\end{array}$ & $\begin{array}{c}\text { Hombres } \\
(\mathbf{n}=\mathbf{8 7})\end{array}$ \\
\hline Edad (años) & $38,8(12,6)$ & $37,3(13,4)$ \\
\hline Peso corporal $(\mathrm{kg})$ & $68,3 \quad(8,6)$ & $74,2 \quad(9,7)$ \\
\hline Talla $(\mathrm{m})$ & $1,55(0,06)$ & $1,65(0,07)$ \\
IMC $\left(\mathrm{kg} / \mathrm{m}^{2}\right)$ & $28,4 \quad(3,4)$ & $27,5 \quad(3,4)$ \\
\hline Estado nutricional $(\%)$ & & \\
Bajo peso & 0 & 1,2 \\
Normal & 17,5 & 24,1 \\
Sobrepeso & 46,9 & 51,7 \\
Obeso & 35,7 & 23,0 \\
\hline
\end{tabular}

Datos presentados como media (desviación estándar) para variables continuas y como \% para variables categóricas. ción de -171,5 min/día en la medición con IPAQ en comparación con la medición registrada con acelerómetro. Para AF total y actividad sedente medidos con cuestionario de autorreporte IPAQ también se observó una subestimación de $-54,8$ y -40,6 min/día en comparación a la medición con acelerómetro, respectivamente (Figura 1). En contraste, la AF moderada y vigorosa fueron sobreestimadas en 91,0 y 47,3 min/día en la medición realizada por cuestionario de autorreporte IPAQ en comparación a la realizada mediante acelerómetro (Tabla 2). Solo la AF moderada presentó una tendencia a disminuir el nivel de sobreestimación. El nivel de sobreestimación se mantuvo constante para AF vigorosa, independiente del tiempo de AF realizado (Figura 1 ).

Al analizar el nivel de discrepancia entre los resultados entregados por cuestionario de autorreporte IPAQ y acelerómetro, según estado nutricional, se observó una subestimación para AF ligera, AF total y tiempo sedente para todas las categorías de estado nutricional (Tabla 3 ). Solo AF total medida con cuestionario de autorreporte IPAQ presentó una tendencia significativa a disminuir el grado de subestimación en la medida que incrementó el IMC, lo que indica que personas con estado nutricional de normopeso presentaron un mayor nivel de subestimación que personas obesas (Figura 2). Tanto AF moderada como AF vigorosa presentaron una sobreestimación de los niveles de AF cuando estos fueron medidos con cuestionario de autorreporte IPAQ, esta sobreestimación fue observada en todas las categorías de estado nutricional (Tabla 3). Solo AF moderada

Tabla 2. Actividad física y actividad sedente según autorreporte (IPAQ) y medición objetiva mediante acelerómetro (ActiGraph)

\begin{tabular}{|lcccc|}
\hline & $\begin{array}{c}\text { IPAQ } \\
\text { (min/día) }\end{array}$ & $\begin{array}{c}\text { Acelerómetro (min/ } \\
\text { día) }\end{array}$ & $\begin{array}{c}\text { Delta IPAQ-Acelerómetro } \\
\text { [95\% IC] }\end{array}$ & Valor p \\
\hline AF ligera & $84,1[73,2 ; 95,0]$ & $255,6[244,4 ; 266,8]$ & $-171,5[-186,5 ;-156,4]$ & $<0,0001$ \\
\hline AF moderada & $126,0[11,4 ; 140,6]$ & $35,0[31,8 ; 38,2]$ & $91,0[75,7 ; 106,2]$ & $<0,0001$ \\
\hline AF vigorosa & $49,6[42,9 ; 56,3]$ & $2,6[1,8 ; 3,3]$ & $47,3[40,3 ; 53,6]$ & $<0,0001$ \\
AF total & $237,2[214,5 ; 259,9]$ & $292,9[281,3 ; 304,5]$ & $-54,8[-79,8 ;-31,5]$ & $<0,0001$ \\
\hline Actividad sedente & $482,1[471,2 ; 493,1]$ & $522,8[512,8 ; 532,8]$ & $-40,6[-44,9 ;-36,4]$ & $<0,0001$ \\
\hline
\end{tabular}

Datos representados como promedio [95\% IC] para niveles de actividad física y tiempo sedente según instrumento de medición. El delta y sus 95\% IC fue calculado mediante [IPAQ-Acelerómetro], por lo cual un delta positivo indica que IPAQ sobreestima los niveles de AF en comparación con acelerómetro, por ende, un delta negativo indica una subestimación del instrumento IPAQ. Para establecer si el delta entre IPAQ y acelerómetro fue significativo se aplicó la prueba de diferencia de medias. 


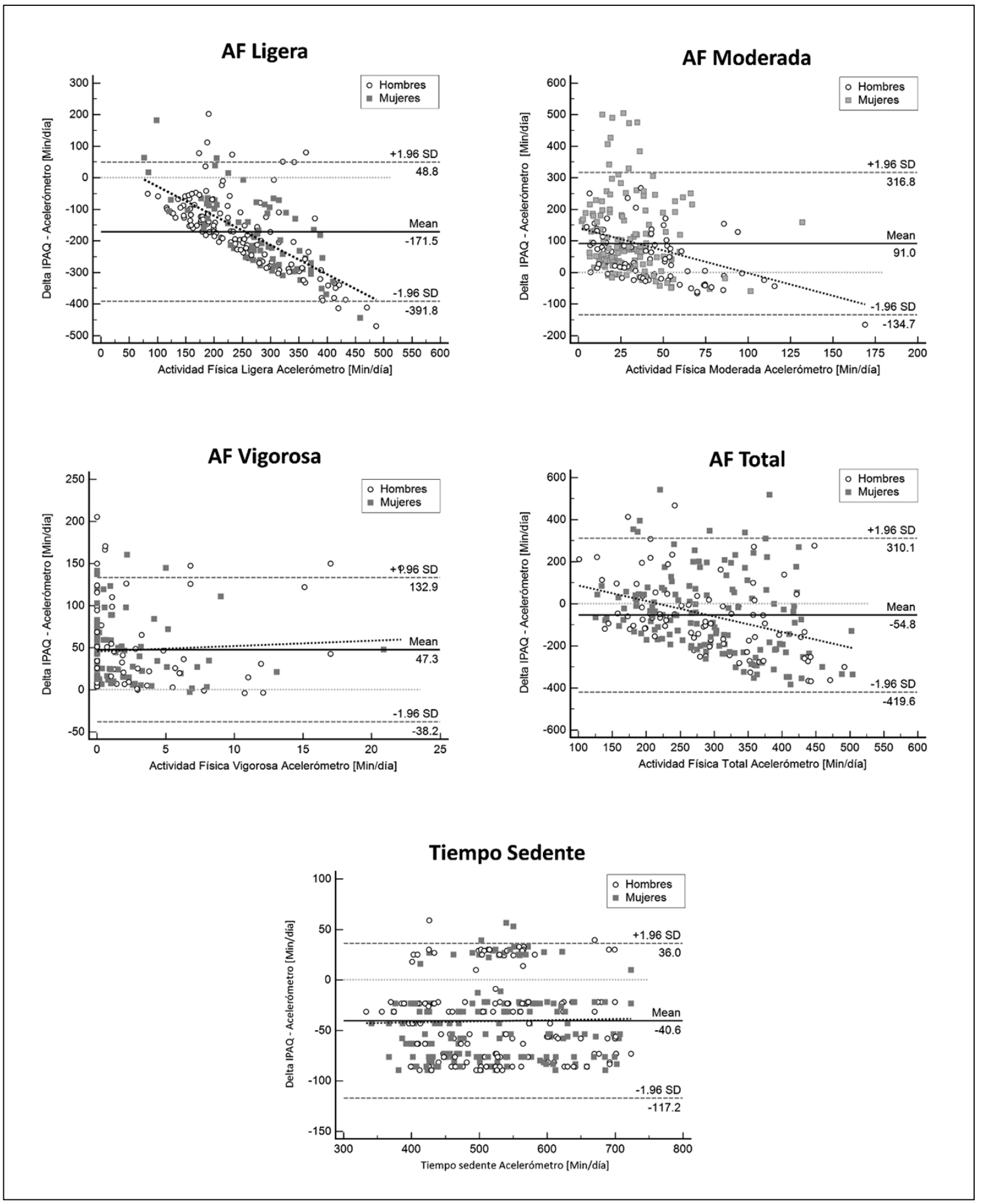

Figura 1. Bland-Altman Plot para diferencias en actividad física y tiempo sedente entre IPAQ y acelerómetros de movimiento. Los datos son presentados como delta entre IPAQ-Acelerómetro, por lo que un delta positivo indica que la medición de IPAQ sobreestima los niveles de actividad física mientras que un valor negativo indica que la medición de IPAQ subestima dichos niveles. La grafica ilustra el promedio para delta (línea horizontal continua —-), 95\% de los márgenes de concordancia (-----). La línea punteada (…...) indica si hubo una tendencia a sobreestimar o subestimar los niveles de actividad física en la medida que incrementa la actividad física medida por acelerómetro. 

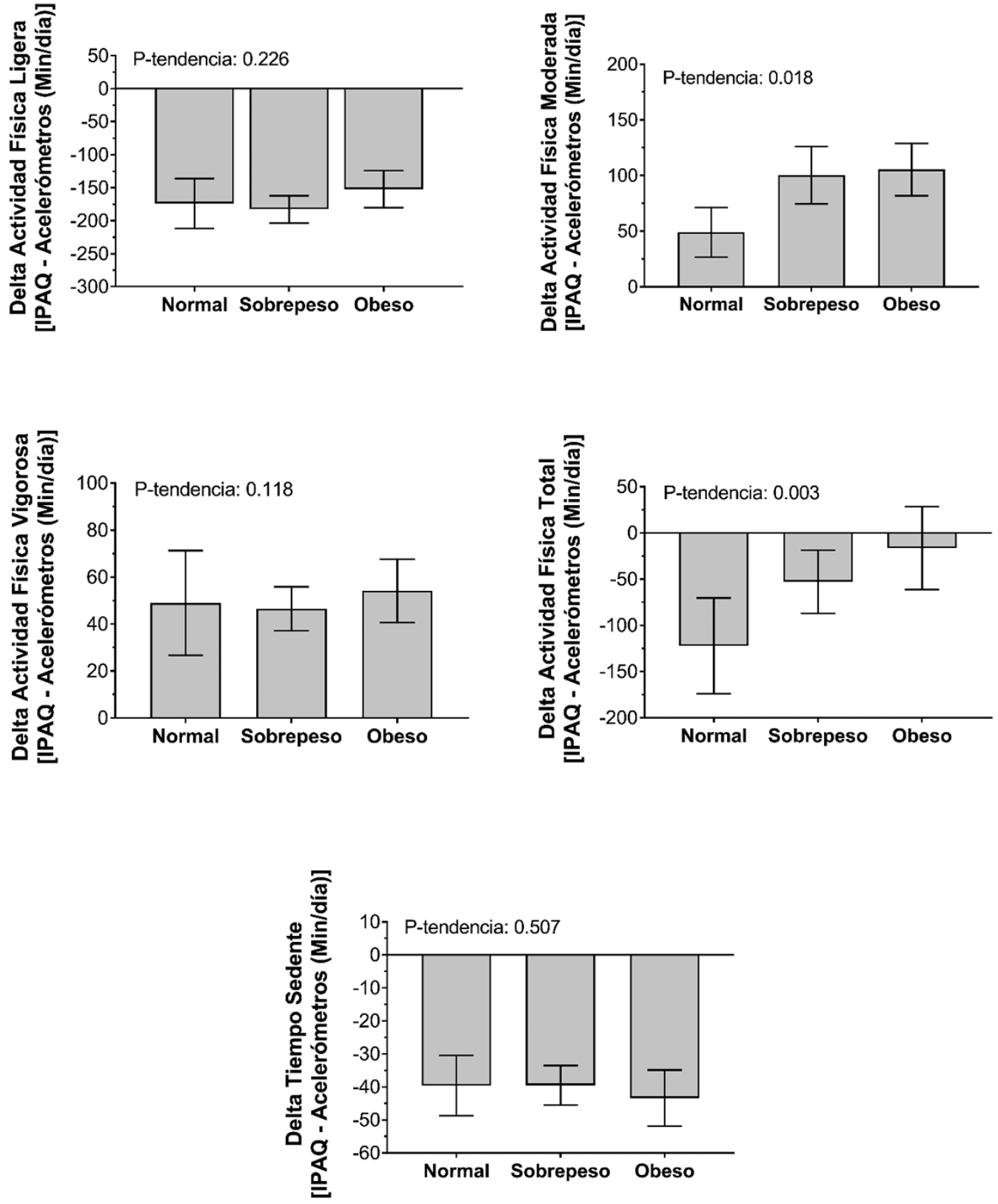

Figura 2. Diferencias en niveles de actividad física y tiempo sedente en mediciones realizadas con IPAQ y acelerómetros de movimiento según estado nutricional. Datos representados como delta en min/día y sus respectivos $95 \%$ IC según estado nutricional. El delta fue calculado mediante [IPAQ-acelerómetro]. Para estimar si las diferencias entre los instrumentos de evaluación presentaron una tendencia significativa a aumentar o disminuir los grados de sobreestimación o subestimaciones según estado nutricional se realizaron análisis de regresión lineales donde estado nutricional fue la variable de exposición codificada de forma ordinal para la estimación del valor p de tendencia. 
Tabla 3. Diferencias entre el autorreporte y la medición objetiva de actividad física a través de acelerómetros de movimiento según estado nutricional

\begin{tabular}{|c|c|c|c|c|}
\hline & $\begin{array}{c}\text { IPAQ } \\
\text { (min/día) }\end{array}$ & $\begin{array}{l}\text { Acelerómetro } \\
\text { (min/día) }\end{array}$ & $\begin{array}{c}\text { Delta IPAQ- Acelerómetro } \\
{[95 \% \text { IC }]}\end{array}$ & $\mathbf{p}$ \\
\hline \multicolumn{5}{|l|}{ AF ligera } \\
\hline Normal & $78,5[51,5 ; 106,0]$ & $252,8[227,9 ; 277,6]$ & $-174,1[-211,8 ;-136,3]$ & $<0,0001$ \\
\hline Sobrepeso & $86,0[71,2 ; 100,9]$ & $268,9[253,3 ; 284,6]$ & $-182,9[-203,6 ;-162,2]$ & $<0,0001$ \\
\hline Obeso & $85,4[64,7 ; 106,2]$ & $237,8[216,5 ; 259,1]$ & $-152,4[-180,4 ;-124,3]$ & $<0,0001$ \\
\hline \multicolumn{5}{|l|}{ AF moderada } \\
\hline Normal & $87,1[65,5 ; 108,8]$ & $38,1[31,3 ; 45,0]$ & $48,9[26,7 ; 71,3]$ & $<0,0001$ \\
\hline Sobrepeso & $137,8[113,1 ; 162,5]$ & $37,5[33,1 ; 41,9]$ & $100,2[74,6 ; 125,9]$ & $<0,0001$ \\
\hline Obeso & $134,9[112,6 ; 157,2]$ & $29,4[23,2 ; 35,6]$ & $105,5[81,9 ; 129,0]$ & $<0,0001$ \\
\hline \multicolumn{5}{|l|}{ AF vigorosa } \\
\hline Normal & $41,6[29,2 ; 54,1]$ & $3,4[0,3 ; 6,4]$ & $38,3[25,2 ; 51,3]$ & $<0,0001$ \\
\hline Sobrepeso & $48,8[39,4 ; 58,2]$ & $2,3[1,5 ; 3,2]$ & $46,5[37,1 ; 55,8]$ & $<0,0001$ \\
\hline Obeso & $56,7[43,0 ; 70,4]$ & $2,7[1,4 ; 3,9]$ & $54,1[40,6 ; 67,6]$ & $<0,0001$ \\
\hline \multicolumn{5}{|l|}{ AF total } \\
\hline Normal & $180,1[135,6 ; 224,7]$ & $302,3[277,6 ; 3,26,9]$ & $-122,1[-173,9 ;-70,4]$ & $<0,0001$ \\
\hline Sobrepeso & $255,2[221,7 ; 288,8]$ & $308,0[291,9 ; 324,1]$ & $-52,8[-86,9 ;-18,6]$ & $<0,0001$ \\
\hline Obeso & $248,9[207,1 ; 290,8]$ & $265,4[243,8 ; 287,1]$ & $-16,5[-61,3 ; 28,4]$ & $<0,0001$ \\
\hline \multicolumn{5}{|c|}{ Actividad sedente } \\
\hline Normal & $454,3[432,2 ; 476,4]$ & $493,9[472,9 ; 514,9]$ & $-39,6[-48,7 ;-30,5]$ & $<0,0001$ \\
\hline Sobrepeso & $468,9[454,3 ; 483,5]$ & $508,4[495,3 ; 521,6]$ & $-39,5[-45,5 ;-33,5]$ & $<0,0001$ \\
\hline Obeso & $522,8[501,6 ; 543,9]$ & $566,1[547,9 ; 584,4]$ & $-43,4[-51,8 ;-34,9]$ & $<0,0001$ \\
\hline
\end{tabular}

Datos representados como promedio en minutos por día y sus respectivos $95 \%$ IC según estado nutricional. El delta fue calculado mediante [IPAQ - acelerómetro] y presentado en minutos/día y sus respectivos 95\% IC. Par estimar si las diferencias entre los instrumentos de evaluación fueron significativamente diferentes según estado nutricional se realizaron análisis de regresión lineales estratificas por estado nutricional.

presentó una tendencia significativa a aumentar el grado de sobreestimación en la medida que se incrementa el IMC, lo que implica que, personas obesas presentaron mayores niveles de sobreestimación que personas con peso normal (Figura 2).

\section{Discusión}

Los principales resultados de este estudio destacan que el cuestionario de autorreporte sobreestimó significativamente la AF moderada y vigorosa, y subestimó la actividad sedente, la AF total y la AF ligera. Este patrón se repitió en todos los estados nutricionales. Además, se observó que personas con estado nutricional normal reportaron mayor precisión en los niveles de AF moderada, pero con menor precisión los niveles de AF total, comparado con el resto de los estados nutricionales. De manera contraria, personas con un estado nutricional clasificado como obesidad fueron más precisas al reportar AF total y menos precisas al reportar AF moderada.

Estos hallazgos son relevantes en términos de salud pública, ya que la medición de AF a nivel nacional ha sido realizada principalmente mediante el uso de cuestionarios de autorreporte ${ }^{3,7,16}$, debido a su bajo costo y fácil aplicación. En este contexto, las prevalencias de AF estimadas a nivel nacional podrían ser imprecisas si se toma en consideración las diferencias que existen entre el autorreporte y la medición objetiva de los niveles de AF mediante acelerómetro. Es por esto que futuros estudios que investiguen los niveles de AF a nivel nacional deberían poner un mayor énfasis en integrar evaluaciones objetivas para comparar 
con las mediciones subjetivas, considerando el importante rol que juega la AF en la prevención de obesidad y de enfermedades crónicas no transmisibles, entre otras ${ }^{17-23}$.

Nuestros resultados concuerdan con evidencia internacional. Al respecto, una revisión sistemática de Skender et al. ${ }^{24}$, que comparaba acelerometría y cuestionarios de auto-reporte de AF, concluyó que los acelerómetros parecen proporcionar resultados más confiables en relación con la AF autorreportada. Sin embargo, debido a la limitada consistencia general y los diferentes aspectos medidos por cada método, se recomienda que los estudios utilicen tanto cuestionarios de autorreporte como acelerómetros para obtener la información más completa sobre la $\mathrm{AF}^{24}$. La sobreestimación de la AF moderada y vigorosa y la subestimación de la actividad sedente también se evidenció en un estudio desarrollado en 6 países (Bélgica, República Checa, Dinamarca, España, Reino Unido y Estados Unidos de Norteamérica) que correlacionó el cuestionario de autorreporte IPAQ en versión completa con mediciones objetivas realizadas con acelerómetro $^{25}$. Nuestros resultados son similares al reporte anterior, ya que los menores deltas corresponden a la AF ligera y sedente. En otro estudio realizado en población chilena, el cuestionario GPAQ de autorreporte mostró validez para medir solo la actividad sedentaria comparando los resultados con mediciones obtenidas con acelerómetro en población chilena ${ }^{26}$.

Además del problema de sobrepeso y obesidad en Chile, la Encuesta Nacional de Salud 2016-2017 muestra que $86,7 \%$ de la población clasifica como sedentaria y que el sedentarismo aumenta con la edad $^{3}$. El presente estudio evidencia la necesidad de medir objetivamente la AF en la población chilena, sobre todo debido a la mayor sobreestimación de la AF moderada reportadas por las personas con estado nutricional de obesidad, ya que este tipo de AF es una de las estrategias recomendadas para combatir el sobrepeso y la obesidad. La información existente en el país en relación a las prevalencias de $\mathrm{AF}^{3,16}$ ha sido derivadas de cuestionarios de autorreporte, los que podrían entregar resultados sesgados debido a la sobreestimación y subestimación del nivel de AF de las personas, ya que, de acuerdo a los resultados reportados en este estudio, varía según estado nutricional.

Según nuestro conocimiento, el presente estudio es el primero en comparar la diferencia entre niveles de AF autorreportados y medición objetiva por acelerometría, según estado nutricional. Una de las fortalezas del presente estudio es la medición con técnicas objetivas estandarizas y cuestionarios de autorreporte de AF validados internacionalmente. Sin embargo, nuestro estudio presenta limitaciones importantes que tienen que ser consideradas al interpretar los resultados de este estudio. La muestra utilizada en este estudio no es representativa de la población chilena lo cual podría haber influenciado las diferencias encontradas entre los métodos para medir AF. Si bien, este estudio incorpora datos de acelerometría como método gold standard, esta metodología también presenta limitaciones, por ejemplo, los acelerómetros de movimiento no capturan cierto tipo de actividades como lo es andar en bicicleta, nadar, realizar ejercicios de resistencia muscular, entre otros, lo cual podría haber influenciado las diferencias encontradas entre métodos.

En conclusión, la medición de AF mediante autorreporte podría presentar importantes niveles de sobreestimación que podrían claramente ocultar los reales niveles de inactividad física de la población chilena, siendo mayor la discrepancia en personas con estado nutricional de obesidad. Contar con una medición más precisa de los niveles de AF es sumamente relevante al momento de definir políticas públicas orientadas a incrementar los niveles de actividad de la población. Por ende, encuestas nacionales realizadas en Chile deberían considerar el uso de metodología objetiva para cuantificar la AF de la población, lo cual permitiría implementar políticas públicas acorde a los verdaderos niveles de inactividad de la población.

\section{Referencias}

1. Manna P, Jain SK. Obesity, Oxidative Stress, Adipose Tissue Dysfunction, and the Associated Health Risks: Causes and Therapeutic Strategies. Metab Syndr Relat Disord 2015; 13 (10): 423-44.

2. WHO. Global Health Observatory (GHO) data. World Health Organization; 2014. https://www.who.int/gho/ en/

3. MINSAL. Encuesta Nacional de Salud 2016-2017. Primeros resultados. Ministerio de Salud (MINSAL). Gobierno de Chile. 2017. https://www.minsal.cl/ 
wp-content/uploads/2017/11/ENS-2016-17_PRIMEROS-RESULTADOS.pdf.

4. Crovetto MM, Uauy R. [Changes in the consumption of dairy products, sugary drinks and processed juices in the Chilean population]. Rev Med Chile 2014; 142 (12): 1530-9.

5. Malo-Serrano M, Castillo N, Pajita D. La obesidad en el mundo. An Fac Med 2017; 173-8.

6. Aguilar Cordero MJ, Sánchez López AM, Barrilao G, R, Rodríguez Blanque R, Noack Segovia J, Pozo Cano MD. Descripción del acelerómetro como método para valorar la actividad física en los diferentes períodos de la vida; revisión sistemática. Nutr Hosp 2014; p. 1250-61.

7. Díaz-Martínez X, Petermann F, Leiva AM, Garrido-Méndez A, Salas-Bravo C, Martínez MA, et al. [Association of physical inactivity with obesity, diabetes, hypertension and metabolic syndrome in the chilean population]. Rev Med Chile 2018; 146 (5): 585-95.

8. Rothney MP, Brychta RJ, Meade NN, Chen KY, Buchowski MS. Validation of the ActiGraph two-regression model for predicting energy expenditure. Med Sci Sports Exerc 2010; 42 (9): 1785-92.

9. Celis-Morales CA, Pérez-Bravo F, Ibáñez L, Salas C, Bailey ME, Gill JM. Objective vs self-reported physical activity and sedentary time: effects of measurement method on relationships with risk biomarkers. PloS one 2012; 7 (5): e36345.

10. Celis-Morales CA, Pérez-Bravo F, Ibanes L, Sanzana R, Hormazabal E, Ulloa N, et al. Insulin Resistance in Chileans of European and Indigenous Descent: Evidence for an Ethnicity x Environment Interaction. PlosOne 2011; 6 (9): e24690.

11. Serón P, Muñoz S, Lanas F. Nivel de actividad física medida a través del cuestionario internacional de actividad física en población chilena. Rev Med Chile 2010; 138 (10): 1232-9.

12. Cristi-Montero C, Ramírez-Campillo R, Álvarez C, Garrido Méndez A, Martínez MA, Díaz Martínez, et al. Fitness cardiorrespiratorio se asocia a una mejora en marcadores metabólicos en adultos chilenos. Rev Med Chile 2016; 144 (8): 980-9.

13. Freedson PS, Melanson E, Sirard J. Calibration of the Computer Science and Applications, Inc. accelerometer. Med Sci Sports Exerc 1998; 30 (5): 777-81.

14. WHO. Obesity: preventing and Managing the Global Epidemic. World Health Organization; 2000.

15. Bland JM, Altman DG. Measuring agreement in method comparison studies. Stat Methods Med Res 1999; 8 (2): 135-60.

16. MINSAL. Encuesta Nacional de Salud 2010. Ministerio de Salud (MINSAL). Gobierno de Chile. 2010. https:// www.minsal.cl/portal/url/item/bcb03d7bc28b64dfe040010165012d23.pdf.

17. Aguilar-Farias N, Martino-Fuentealba P, Cortinez-O'Ryan A, Chandia-Poblete D, Celis-Morales CA, Bahamondes $\mathrm{P}$, et al. The descriptive epidemiology of sitting in Chilean adults: Results from the National Health Survey 2009-2010. J Sport Health Sci 2019; 8 (1): 32-8.

18. Leiva AM, Petermann-Rocha F, Martínez-Sanguinetti MA, Troncoso-Pantoja C, Concha Y, Garrido-Méndez A, et al. [Association of a healthy lifestyle index with cardiovascular risk factors in Chileans adults]. Rev Med Chile 2018; 146 (12): 1405-14.

19. Troncoso C, Petermann-Rocha F, Brown R, Leiva AM, Martínez MA, Díaz-Martínez X, et al. Patterns of healthy lifestyle behaviours in older adults: Findings from the Chilean National Health Survey 2009-2010. Exp Gerontol 2018; 113: 180-5.

20. Cristi-Montero C, Steell L, Petermann F, Garrido-Méndez A, Díaz-Martínez X, Salas-Bravo C, et al. Joint effect of physical activity and sedentary behaviour on cardiovascular risk factors in Chilean adults. J Public Health (Oxf) 2018; 40 (3): 485-92.

21. Díaz-Martínez X, Steell L, Martínez MA, Leiva AM, Salas-Bravo C, Labrana AM, et al. Higher levels of self-reported sitting time is associated with higher risk of type 2 diabetes independent of physical activity in Chile. J Public Health (Oxf) 2018; 40 (3): 501-7.

22. Steell L, Garrido-Méndez A, Petermann F, Díaz-Martínez X, Martínez MA, Leiva AM, et al. Active commuting is associated with a lower risk of obesity, diabetes and metabolic syndrome in Chilean adults. J Public Health (Oxf) 2018; 40 (3): 508-16.

23. Álvarez C, Ramírez-Campillo R, Martínez-Salazar C, Mancilla R, Flores-Opazo M, Cano-Montoya J, et al. Low-Volume High-Intensity Interval Training as a Therapy for Type 2 Diabetes. Int J Sports Med 2016; 37 (9): 723-9.

24. Skender S, Ose J, Chang-Claude J, Paskow M, Brühmann B, Siegel EM, et al. Accelerometry and physical activity questionnaires-a systematic review. BMC Public Health 2016; 16: 515.

25. Cerin E, Cain KL, Oyeyemi AL, Owen N, Conway TL, Cochrane T, et al. Correlates of Agreement between Accelerometry and Self-reported Physical Activity. Med Sci Sports Exerc 2016; 48 (6): 1075-84.

26. Aguilar-Farias N, Leppe Zamora J. Is a single question of the Global Physical Activity Questionnaire (GPAQ) valid for measuring sedentary behaviour in the Chilean population? J Sports Sci 2017; 35 (16): 1652-7. 